H. Kaneta

Nagoya Math. J.

Vol. 57 (1975), 87-106

\title{
ON THE ASYMPTOTIC BEHAVIOUR OF A DIFFUSION PROCESS WITH SINGULAR DRIFT
}

\author{
HITOSHI KANETA
}

We discuss some peculiar features of the diffusion process whose characterization is given below. Let $D$ be a bounded domain in the $d$ dimensional Euclidean space $E^{d}$ with a smooth boundary $\partial D$. The domain $D$ contains open balls $C_{i}^{\prime} s(i=1, \ldots, n)$ which are mutually disjoint. Our process is a diffusion process on the state space $D \cup \partial D$ which is locally equivalent to the Brownian motion except on the spheres $\partial C_{i}^{\prime} s$ and the boundary $\partial D$. By a diffusion process we mean a continuous strong Markov process. As to the terminology about Markov processes we refer to [2]. Let the ratio of probabilities for the tragectory to go into and out of the ball $C_{i}$ be $a c_{i}^{-1}$, while $\partial D$ is assumed to be a reflecting barrier. Although the existence of such a process is not obvious, it is guaranteed. We are mainly interested in how the local behaviour around $\partial C_{i}^{\prime} s$ determines the asymptotic behaviour of the process on the whole space. It is proved that such a process has the unique invariant measure to which the transition probability converges in the sense of the total variation as time goes by. It seems very likely that we can construct such a process and that the same result holds even when $\partial C_{i}^{\prime} s$ are taken to be any smooth hypersurfaces. In such a case we can appeal to the theory of Dirichlet space discussed by M. Fukushima.

For the precise definition of the process we refer to Section 1, where we state our main theorems. In Sections 2 and 3 we prepare some properties of the auxiliary processes. Using the results in these sections we prove our theorems in Sections 4 and 5 .

The author expresses his sincere thanks to Professor I. Kubo and Professor $\mathrm{H}$. Kunita for their valuable suggestions and kind encouragement.

Received February 22, 1974. 


\section{Main theorems}

In this section we represent two theorems. To begin with we prepare some definitions and notations. When $E$ denotes a semi-compact space, $\mathscr{B}(E), B(E), C(E)$ and $\hat{C}(E)$ stand for the topological $\sigma$-algebra, all realvalued bounded $\mathscr{R}(E)$-measurable functions, all real-valued bounded continuous functions and all real-valued continuous functions vanishing at infinity, respectively. As to the $\sigma$-algebras attached to a Markov process we always consider the smallest one unless otherwise stated. We define a part process of a given Markov process on $E$. Let $X=\left(\Omega, x_{t}, \zeta, N_{t}\right.$, $N, P_{x}$ ) be a right-continuous strong Markov process on $E$ and let $F$ be a non-empty open subset such that either the closure $\bar{F}$ or the complement $F^{c}$ is compact. We set

$$
\begin{aligned}
\tau & =\text { the first exit time from } F, \\
\tilde{\Omega} & =\{\tau>0\}, \\
\tilde{N}_{t} & =N_{t}\{\tau>t\}, \\
\tilde{N} & =N\{\tau>0\} .
\end{aligned}
$$

As is known, $X_{F}=\left(\tilde{\Omega}, x_{t}, \tau, \tilde{N}_{t}, \tilde{N}, P_{x}\right)$ is a right-continuous strong Markov process on the subset $F$ (Theorem 10.1 in [2]). We call this process the $F$ part of the process $X$ and denote it by $X_{F}$.

We are mainly concerned with a bounded domain $D$ in the $d$-dimensional Euclidean space $E^{d}$ with a smooth boundary $\partial D$. We fix the domain $D$ and divide it into mutually disjoint sets $A, C_{i}$ and $\partial C_{i}$ $(i=1, \cdots, n) . \quad C_{i}^{\prime} s$ are open balls with radius $\xi_{i}$ centered at $\varepsilon_{i}$ respectively and $\partial C_{i}^{\prime} s$ denote their boundaries i.e. spheres. Let $B_{i}(i=1, \cdots, n)$ be the open ball with radius $r_{i}\left(>\xi_{i}\right)$ centered at $\varepsilon_{i}$ such that the closures $\bar{B}_{i}^{\prime} s$ are mutually disjoint and contained in the domain $D$. Finally we set $B_{0}=A \cup \partial D, \partial B=\bigcup_{i=1}^{n} \partial B_{i}, \partial C=\bigcup_{i=1}^{n} \partial C_{i}$ and $\bar{D}=D \cup \partial D$. See Figures 1 and 2, where broken lines denote $\partial B_{i}^{\prime} s$.

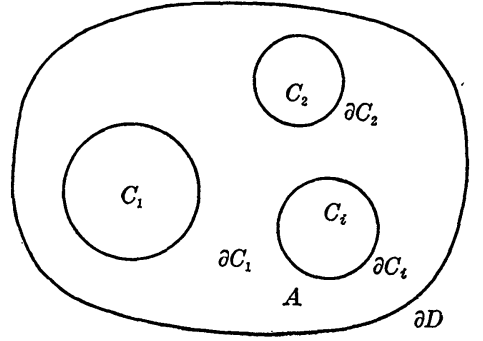

Figure 1

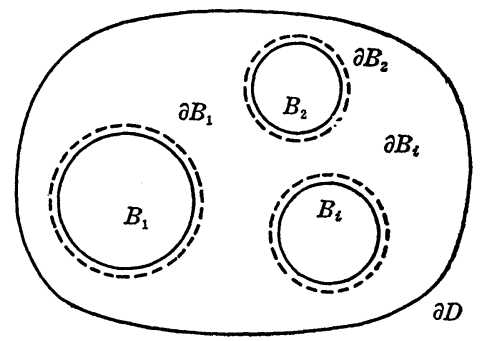

Figure 2 
Now we introduce two kinds of diffusion processes from which we construct our desired diffusion process on $\bar{D}$. The first is the reflecting barrier Brownian motion $X^{0}=\left(x_{t}, P_{x}^{0}\right)$ on $\bar{D}$. Of course $X^{0}$ is the process whose transition density with respect to the Lebesgue measure is equal to the fundamental solution of a heat equation on $\bar{D}$;

$$
\left(\frac{\partial}{\partial t}-\Delta\right) u(t, x)=0 \text {, }
$$

with boundary condition

$$
\frac{\partial u}{\partial n}(t, x)=0 \quad x \in \partial D
$$

The second is the process $X^{i}=\left(x_{t}, P_{x}^{i}\right)(i=1, \cdots, n)$ on $B_{i} \cup \partial B_{i}$ which is expressible as a skew product of a process $R^{i}=\left(r_{t}, P_{r}^{i}\right)$ on the closed interval $\left[0, r_{i}\right]$ and the spherical Brownian motion $\Theta=\left(\theta(t), P_{\theta}\right)$ on the unit sphere $S^{d-1}$, where the process $R^{i}$ denotes the non-singular conservative diffusion process possessing the following speed measure $d m(r)$ and the scale measure $d s(r)$ with a reflecting barrier $r_{i}$;

$$
\begin{aligned}
& d m(r)=a^{-1} r^{d-1} d r, \quad d s(r)=a r^{-d+1} d r \quad\left(0<r<\xi_{i}\right), \\
& d m(r)=c_{i}^{-1} r^{d-1} d r, \quad d s(r)=c_{i} r^{-d+1} d r \quad\left(\xi_{i} \leq r<r_{i}\right),
\end{aligned}
$$

where $a$ and $c_{i}^{\prime} s$ are given positive constants. We note that the process $R^{i}$ depends only on the ratio $a c_{i}^{-1}$. As the additive functional $\ell_{t}$ we take $\int_{0}^{t} r_{s}^{-2} d s$. Here the differential generator of the spherical Brownian motion is the spherical Laplace operator. For the details about the skew product we refer to 7.16 in [6].

Now we are ready to state our results.

THEOREM 1. There exists a diffusion process $\hat{X}=\left(X_{t}, \infty, N_{t}, N, P_{x}\right)$ on the compact set $\bar{D}$ such that the part process $\hat{X}_{B_{i}}$ is equivalent to the part process $X_{B_{i}}^{i}$ for every $i(i=0,1, \cdots, n)$. All right-continuous strong Markov processes on $\bar{D}$ with such a property are mutually equivalent and induce a continuous Feller semi-group on $C(\bar{D})$.

Let $X^{\cdot}=\left(x_{t}^{\cdot}, P_{x}^{\cdot}\right)$ be the $d$-dimensional Brownian motion. As will be shown in Lemmas 2.1 and 3.1, the part processes $X_{A}^{0}$ and $X_{C_{i}}^{i}$ $(i=1, \cdots, n)$ are equivalent to $X_{\dot{A}}^{\cdot}$ and $X_{C_{i}}$ respectively. Thus the part processes $\hat{X}_{A}$ and $\hat{X}_{C_{i}}$ of $\hat{X}$ in Theorem 1 are equivalent to $X_{A}^{\cdot}$ and $X_{C_{i}}^{\dot{C}}$ 
respectively. In other words, the process $\hat{X}$ is locally equivalent to the Brownian motion except on $\partial C$ and $\partial D$. To describe the behaviour of tragectories starting from $\partial C_{i}$, we introduce two spheres. Let $S_{+\delta}$ (resp. $S_{-\delta}$ ) be the sphere with radius $\xi_{i}+\delta$ (resp. $\xi_{i}-\delta$ ) centered at $\varepsilon_{i}$. If we denote by $\tau_{\delta}$ the first exit time from the domain limited by the two spheres, we have for all $x \in \partial C_{i}$

$$
\begin{aligned}
& \lim _{\delta \rightarrow 0} P_{x}\left[X_{\tau \delta} \in S_{-\delta}\right]=\frac{c_{i}}{a+c_{i}}, \\
& \lim _{\delta \rightarrow 0} P_{x}\left[X_{\tau \delta} \in S_{+\delta}\right]=\frac{a}{a+c_{i}},
\end{aligned}
$$

because the radial part of $X^{i}$ has the following scale measure $d s(r)$;

$$
d s(r)=a r^{-d+1} \quad\left(0<r<\xi_{i}\right), \quad d s(r)=c_{i} r^{-d+1} d r \quad\left(\xi_{i} \leq r<r_{i}\right) .
$$

Finally we remark that the process $\hat{X}$ depends only on spheres $\partial C_{i}^{\prime} s$ and the ratios $a c_{i}^{-1} s$, but not on the diameters $r_{i}^{\prime} s$ of $B_{i}^{\prime} s$, because the characteristic operator of $\hat{X}$ does not depend on the choice of $B_{i}^{\prime}$ s.

Before stating Theorem 2 we consider the non-singular conservative diffusion process on the closed interval $[0,1]$ corresponding to the speed measure $d m(r)$ and the scale measure $d s(r)$ given below with the reflecting barriers;

$$
\begin{aligned}
& d m(r)=a^{-1} d r, \quad d s(r)=a d r \quad(0<r<\xi), \\
& d m(r)=c^{-1} d r, \quad d s(r)=c d r \quad(\xi \leq r<1),
\end{aligned}
$$

where $a, c$ and $\xi$ are given positive constants. As is known, this process is locally equivalent to the one-dimensional Brownian motion on $(0, \xi)$ and on $(\xi, 1)$. At the exceptional point $\xi$, the same relations as (1.2) hold if we replace $S_{+\delta}$ and $S_{-\delta}$ by $\xi+\delta$ and $\xi-\delta$, respectively. Furthermore the transition probability converges to the unique invariant probability measure which is equal to the speed measure up to a constant multiplication ([6] or [7] and Lemma 5.3). This fact admits the following physical interpretation. If we suspend particles moving according to the diffusion law into a vessel $[0,1]$, the vessel will be separated into two parts $(0, \xi)$ and $(\xi, 1)$. In $(0, \xi)$ the density of particles is to be equal to $c$ and in $(\xi, 1)$ to $a$. We can expect that the process $\hat{X}$ in Theorem 1 also exhibits the same phenomenon due to the singularity on $\partial C$ expressed in (1.2). 
THEOREM 2. The process $\hat{X}$ specified in Theorem 1 has a unique invariant measure $\mu$;

$$
\mu(d x)=c\left(a l_{A}(x)+\sum_{i=1}^{n} c_{i} l_{c_{i}}(x)\right) d x,
$$

where $a$ and $c_{i}^{\prime}$ s are constants appearing in (1.1), $c$ being the normalization constant. Let $\nu$ be any initial distribution and $P_{t}(\nu, \cdot)$ be the transition probability of the process with the initial distribution $\nu$. Then we have

$$
\lim _{t \rightarrow \infty}\left\|P_{t}(\nu, \cdot)-\mu(\cdot)\right\|=0,
$$

where \|\| means the total variation. If $f \in B(\bar{D})$, for all $x \in \bar{D}$ we have

$$
P_{x}\left[\lim _{t \rightarrow \infty} \frac{1}{t} \int_{0}^{t} f\left(X_{s}\right) d s=\int_{D} f d \mu\right]=1 .
$$

We will prove these theorems in Sections 4 and 5. Sections 2 and 3 are devoted to prepare some properties of processes $X^{i}(i=0,1, \cdots, n)$. The reader is recommended to read Sections 4 and 5 first, consulting Sections 2 and 3 if necessary.

\section{Some properties of the process $X^{0}$}

In this section we exhibit simple properties of the reflecting barrier Brownian motion $X^{0}$ on $\bar{D}$. We denote the process by $X=\left(x_{t}, P_{x}\right)$ instead of $X^{0}=\left(x_{t}, P_{x}^{0}\right) . \quad X^{\cdot}=\left(x_{t}^{*}, P_{x}^{\cdot}\right)$ stands for the $d$-dimensional Brownian motion whose differential generator is the Laplace operator $\Delta$. As is shown in [11], $X$ is a strong Feller process inducing a continuous Feller semi-group on $C(\bar{D})$. Moreover the transition probability $\left\{P_{t}(x, \cdot)\right\}$ satisfies

$$
\sup _{x \in \bar{D}} P_{t}(x, U(\varepsilon, x))=o(t) \quad(\text { for small } t),
$$

where $U(\varepsilon, x)$ denotes the $\varepsilon$-neighbourhood of $x$.

LEMMA 2.1. The part process $X_{D}$ is equivalent to $X_{D}^{\cdot}$.

Proof. As is shown in [9], the transition density of the part process $X_{D}$ is equal to the fundamental solution of a heat equation,

$$
\left(\frac{\partial}{\partial t}-\Delta\right) u(t, x)=0
$$


with boundary condition

$$
u(t, x)=0 \quad x \in \partial D .
$$

The same is true for the part process $X_{D}^{\circ}$ (Theorem 13.22 in [2]). From the uniqueness of the fundamental solution the lemma follows. Q.E.D.

LEMMA 2.2. Let $\tau$ be the first exit time from $B_{0}$ and $K$ be a compact set contained in $B_{0}$. Then if $t$ is positive, we have

(1) $\sup _{x \in K} P_{x}[\tau \leq t]<1$,

(2) $\sup _{x \in K} P_{x}[\tau \leq t]=o(t) \quad$ (for small $t$ ),

(3) $\inf _{x \in K} P_{x}[\tau \leq t]>0$,

(4) $E_{x}[\tau]<\infty \quad\left(x \in B_{0}\right)$.

Proof. From Theorem 13.7 in [2], (4) follows. Let $K_{i}(i=1, \cdots, n)$ be an open ball such that $C_{i} \subset K_{i} \subset B_{i}$ with the common center $\varepsilon_{i}$ and that $K \subset \bigcap_{i=1}^{n} K_{i}^{c}$. We denote by $\tau^{\prime}$ the first exit time from the interior of $\bigcap_{i=1}^{n} K_{i}^{c}$ and set $\partial K=\bigcup_{i=1}^{n} \partial K_{i}$. Theorem 13.1 in [2] tells us tinuity of $P_{x}[\tau \leq t]$ on $B_{0}$. Thus if $x \in K$, it follows that

$$
\begin{aligned}
P_{x}[\tau \leq t] & =P_{x}\left[\tau^{\prime}+\theta_{\tau^{\prime}} \tau \leq t\right] \leq P_{x}\left[\theta_{\tau^{\prime}} \tau \leq t\right] \\
& =E_{x}\left[P_{x_{\tau^{\prime}}}[\tau \leq t]\right] \leq \max _{x \in \partial K} P_{x}[\tau \leq t] .
\end{aligned}
$$

Since $X$ is locally equivalent to the Brownian motion on $D$, (2) follows. Since $P_{x}[\tau \leq t]$ is positive and continuous on $B_{0}$, (3) follows. I. V. Girsanov has shown in [3] that

$$
\sup _{x \in K} P_{x}[\tau \leq t]=0(t)
$$

under the condition

$$
\sup _{x \in \bar{D}} P_{t}(x, U(\varepsilon, x))=0(t) .
$$

We can replace $0(t)$ by $o(t)$ on account of (2.1).

Q.E.D.

LEMMA 2.3. Let $U$ be a non-empty open set in $\partial C$ and let $\tau$ be the first exit time from $B_{0}$. Then $P_{x}\left[\tau \leq t, x_{\tau} \in U\right]$ is positive, if $x \in B_{0}$ and $t$ is positive.

Proof. We denote by $U(\delta, y)$ the $\delta$-neighbourhood of $y$. For a point $y \in U$, there exists $\delta$ such that $(U(\delta, y) \cap \partial C) \subset U$. We set $U^{\prime}=U\left(\frac{1}{3} \delta, y\right)$ 
$\cap A$. To every point $x^{\prime} \in U^{\prime}$ we attach $U\left(\frac{2}{3} \delta, x^{\prime}\right)$ with the boundary $S\left(x^{\prime}\right)$ and let $\tau^{\prime}$ be the first exit time from $U\left(\frac{2}{3} \delta, x^{\prime}\right)$. Recall $C=\bigcup_{i=1}^{n} C_{i}$. If $t-s$ is positive, it holds that

$$
P_{x^{\prime}}\left[\tau \leq t-s, x_{\tau} \in U\right] \geq P_{x^{\prime}}\left[\tau^{\prime} \leq t-s, x_{\tau^{\prime}} \in S\left(x^{\prime}\right) \cap C\right]>0 .
$$

The last inequality holds because the process $X$ is locally equivalent to the Brownian motion on $D$. On the other hand, $P_{x}\left[x_{s} \in U^{\prime}, s<\tau\right]$ is positive whenever $x \in B_{0}$ (cf. Lemma 2.5). Thus if $x \in B_{0}$, it follows that

$$
\begin{aligned}
P_{x}\left[\tau \leq t, x_{\tau} \in U\right] & \geq P_{x}\left[x_{s} \in U^{\prime}, s<\tau, \tau \leq t, x_{\tau} \in U\right] \\
& =P_{x}\left[x_{s} \in U^{\prime}, s<\tau, \theta_{s}\left\{\tau \leq t-s, x_{\tau} \in U\right\}\right] \\
& =E_{x}\left[P_{x_{s}}\left[\tau \leq t-s, x_{s} \in U\right]: x_{s} \in U^{\prime}, s<\tau\right]>0 .
\end{aligned}
$$

Q.E.D.

LEMMA 2.4. Let $\tau$ be the first exit time from $B_{0}$. If $f \in B\left(B_{0}\right)$ and $\alpha$ is positive, the function $x \rightarrow E_{x}\left[\int_{0}^{\tau} e^{-\alpha t} f\left(x_{t}\right) d t\right]$ belongs to $C\left(B_{0}\right)$. If $g \in B(\partial C)$ and $\alpha$ is non-negative, the function $x \rightarrow E_{x}\left[e^{-\alpha \tau} g\left(x_{\tau}\right)\right]$ also belongs to $C\left(B_{0}\right)$.

Proof. According to Theorem 13.1 in [2], the function $x \rightarrow E_{x}\left[f\left(x_{t}\right)\right.$ : $t<\tau]$ is continuous on $B_{0}$. So the first assertion follows at once. We note that

$$
\begin{aligned}
E_{x}\left[e^{-\alpha \tau} g\left(x_{\tau}\right)\right]= & E_{x}\left[e^{-\alpha \tau} g\left(x_{\tau}\right): t<\tau\right]+E_{x}\left[e^{-\alpha \tau} g\left(x_{\tau}\right): \tau \leq t\right] \\
= & E_{x}\left[\theta_{t}\left\{e^{-\alpha(\tau+t)} g\left(x_{\tau}\right): t<\tau\right]+E_{x}\left[e^{-\alpha \tau} g\left(x_{\tau}\right): \tau \leq t\right]\right. \\
= & e^{-\alpha t} E_{x}\left[E_{x_{t}}\left[e^{-\alpha \tau} g\left(x_{\tau}\right)\right]\right]-e^{-\alpha t} E_{x}\left[E_{x_{t}}\left[e^{-\alpha \tau} g\left(x_{\tau}\right)\right]: \tau \leq t\right] \\
& +E_{x}\left[e^{-\alpha \tau} g\left(x_{\tau}\right): \tau \leq t\right] .
\end{aligned}
$$

The first term on the right of the last equality is continuous because $X$ is a strong Feller process. Applying (2) in Lemma 2.2 to the other terms, we see that the term on the left is continuous on $B_{0}$. Q.E.D.

LEMMA 2.5. The transition probability of the part process $X_{B_{0}}$ is equivalent to the Lebesgue measure.

Proof. Apparently the transition probability is absolutely continuous with respect to the Lebesgue measure. Lemma 2.1 implies that the part process $X_{A}$ is equivalent to $X_{A}^{\cdot}$. By (1) in Lemma 2.2, $P_{x}[t<\tau]$ is positive if $x \in \partial D$. From these facts the converse also holds. Q.E.D. 
LEMMA 2.6. If $f \in B\left(B_{0}\right)$ and $f$ is non-negative, we have

$$
\int_{B_{0}} E_{x}\left[f\left(x_{t}\right): t<\tau\right] d x \leq \int_{B_{0}} f d x
$$

where $\tau$ denotes the first exit time from $B_{0}$.

Proof. Defining $f(x)=0$ on $B_{0}^{c}$, we assume $f \in B(\bar{D})$. Since the Lebesgue measure is an invariant measure of the process $X$, it follows that

$$
\int_{B_{0}} E_{x}\left[f\left(x_{t}\right): t<\tau\right] d x \leq \int_{D} E_{x}\left[f\left(x_{t}\right)\right] d x=\int_{D} f d x=\int_{B_{0}} f d x .
$$

Q.E.D.

\section{Some properties of the process $X^{i}$}

In this section we exhibit some properties of the process $X^{i}(i=1$, $\cdots, n)$ on the state space $\bar{B}_{i}=B_{i} \cup \partial B_{i}$. We may restrict our attention to one of them, say to the $i$-th process $X^{i}$, and denote it by $X=\left(x_{t}, P_{x}\right)$. For the simplicity of notations $B, \partial B, C$ and $R=\left(r_{t}, P_{r}\right)$ stand for $B_{i}$, $\partial B_{i}, C_{i}$ and the process $R^{i}=\left(r_{t}, P_{r}^{i}\right)$, which is the radial part of $X^{i}$. We denote the state space of $R$ by $[0, q]$ in place of $\left[0, r_{i}\right]$. Finally $\Theta=$ $\left(\theta(t), P_{\theta}\right)$ stands for the spherical Brownian motion on the unit sphere $S^{d-1}$ in the $d$-dimensional Euclidean space $E^{d}$. Its transition density $p\left(t, \theta, \theta^{\prime}\right)$ has the following form;

$$
P\left(t, \theta, \theta^{\prime}\right)=\sum_{n=0}^{\infty} e^{-\gamma_{n} t} \sum_{\ell} S_{n}^{\ell}(\theta) S_{n}^{\ell}\left(\theta^{\prime}\right)
$$

where $\gamma_{n}=n(n+d-2)$ and where $\left\{S_{n}^{\ell}(\theta)\right\}$ denote the spherical harmonics of weight $n$. Recall that the Bessel process $R^{\cdot}=\left(r_{t}^{*}, P_{r}^{\cdot}\right)$ on $[0, \infty)$ is the conservative non-singular diffusion process with speed measure $d m^{*}(r)$ and scale measure $d s^{\circ}(r)$ as follows;

$$
d m \cdot(r)=r^{d-1} d r, \quad d s^{\bullet}(r)=r^{-d+1} d r .
$$

Consider an additive functional $\ell_{t}=\int_{0}^{t} r_{s}^{-2} d s$. As is well known, the skew product $X^{\cdot}=\left(\left[r_{t}^{*}, \theta\left(\ell_{i}\right)\right], P_{r}^{\cdot} \times P_{\theta}\right)$ is equivalent to the Brownian motion on $E^{a}$. To be more precise, sample paths starting from the origin must be added (see 7.16 in [6]).

LEMMA 3.1. The part process $X_{A \cap B}$ is equivalent to $X_{A \cap B}$, so is $X_{C}$ to $X_{C}^{\cdot}$. 
Proof. The part process $R_{(\xi, q)}$ is equivalent to $R_{(\xi, q)}$ because both processes induce continuous semi-groups on $\hat{C}((\xi, q))$ and their infinitesimal operator coincide. Hence

$$
P_{r}\left[r_{t} \in d r, \ell_{t} \in d u, t<\tau\right]=P_{r}^{*}\left[r_{t}^{*} \in d r, \ell_{t} \in d u, t<\tau^{*}\right],
$$

where $\tau$ and $\tau^{\cdot}$ denote the first exit times from $(\xi, q)$. For $f \in B((\xi, q))$ and $g \in B\left(S^{d-1}\right)$ it holds that

$$
\begin{aligned}
E_{r} \times E_{\theta}\left[f\left(r_{t}\right) g\left(\theta\left(\ell_{t}\right)\right): t<\tau\right] & =E_{r}\left[f\left(r_{t}\right) \int_{S^{d-1}} p\left(\ell_{t}, \theta, \theta^{\prime}\right) g\left(\theta^{\prime}\right) d \theta^{\prime}: t<\tau\right] \\
& =E_{r}^{*} \times E_{\theta}\left[f\left(r_{t}^{*}\right) g\left(\theta\left(\ell_{t}^{\prime}\right)\right): t<\tau^{*}\right] .
\end{aligned}
$$

This implies the equivalence of $X_{A \cap B}$ and $X_{A \cap B}$. The proof of the second assertion of the lemma is the same.

Throught the rest of this section, $\sigma$ and $\tau$ mean the first exit times from $B$ and from $[0, q)$ respectively.

LEMMA 3.2. Let $K$ be a compact set contained in $B$. We have for positive $t$

(1) $\sup _{x \in K} P_{x}[\sigma \leq t]>0$,

(2) $\sup _{x \in K} P_{x}[\sigma \leq t]=o(t) \quad$ (for small $t$ ),

(3) $\inf _{x \in K} P_{x}[\sigma \leq t]>0$,

(4) $E_{x}[\sigma]<\infty$.

Proof. Consider the diffusion process $R^{*}=\left(r_{t}^{*}, P_{r}^{*}\right)$ on $[0, \infty)$ whose speed measure $d m(r)$ and scale measure $d s(r)$ are

$$
\begin{array}{llll}
d m(r)=a^{-1} r^{d-1} d r, & d s(r)=a r^{-d+1} d r & & (0<r<\xi), \\
d m(r)=c^{-1} r^{d-1} d r, & d s(r)=c r^{-d+1} d r & & (\xi \leq r<\infty) .
\end{array}
$$

That is, the speed and scale measures of the process $R$ are equal to the restrictions on $(0, q)$ of the speed and scale measures of the process $R^{*}$. We denote by $\tau^{*}$ the first exit time from $[0, q)$. Since the transition functions of $R^{*}$ and $R_{[0, q)}^{*}$ have positive densities with respect to the speed measure $d m(r)$ [6], it is easy to see that

$$
0<P_{0}^{*}\left[\tau^{*} \leq t\right] \leq P_{r}^{*}\left[\tau^{*} \leq t\right]<1 \quad(r<q) .
$$

On account of D. B. Ray's estimate we see $P_{r}^{*}\left[\tau^{*} \leq t\right]=o(t)$, if $r<q$ [6]. Let $M_{a}$ and $M_{b}$ the first hitting times to $a$ and to $b(0<a<r<$ 
$b=q$ ) respectively. Define a symmetric function $G_{a b}\left(r, r^{\prime}\right)$ on $[a, b] \times$ $[a, b]$ by

$$
G_{a b}\left(r, r^{\prime}\right)=\frac{(s(r)-s(a))\left(s(b)-s\left(r^{\prime}\right)\right)}{s(b)-s(a)} \quad\left(r \leq r^{\prime}\right)
$$

where $s(r)=\int^{r} d s\left(r^{\prime}\right)$. As is well known, it holds that

$$
E_{r}^{*}\left[M_{a} \wedge M_{b}\right]=\int_{a}^{b} G_{a b}\left(r, r^{\prime}\right) d m\left(r^{\prime}\right)
$$

Since $r=0$ is a non-exit barrier, $\lim _{a \rightarrow 0} M_{a} \wedge M_{b}=M_{b}$. A simple calculation tells us that (3.1) is dominated by $F(b)$ which does not depend on $a$ nor $r(0<r<b=q)$. Hence $E_{r}^{*}\left[\tau^{*}\right]$ is finite, if $r$ is positive. We note that

$$
E_{0}^{*}\left[\tau^{*}\right] \leq E_{0}^{*}\left[t+\theta_{t} \tau^{*}\right]=t+E_{0}^{*}\left[E_{r_{t}^{*}}^{*}\left[\tau^{*}\right]\right] \leq t+F(b)<\infty,
$$

since $r=0$ is an entrance barrier. Now we observe that the part process $R_{[0, q)}^{*}$ is equivalent to $R_{[0, q)}$. This completes the proof. Q.E.D.

LEMMA 3.3. Let $U$ be a non-empty open set in $\partial B$. If $t$ is positive, $P_{x}\left[\sigma \leq t, x_{\sigma} \in U\right]$ is also positive for all $x \in B$.

Proof. If $x=(0, \theta)$, it is easy to see that

$$
P_{x}\left[\sigma \leq t, x_{\sigma} \in U\right]=P_{0}[\tau \leq t] \int_{S^{d-1}} l_{U}(\theta) d \theta>0 .
$$

For $x=(r, \theta)$ with $0<r<q$, it holds that

$$
\begin{aligned}
P_{x}\left[\sigma \leq t, x_{\sigma} \in U\right] & =P_{r} \times P_{\theta}\left[\tau \leq t, \theta\left(\ell_{\tau}\right) \in U\right] \\
& =E_{r}\left[\int_{S^{d-1}} p\left(\ell_{\tau}, \theta, \theta^{\prime}\right) l_{U}\left(\theta^{\prime}\right) d \theta^{\prime}: \tau \leq t\right]>0,
\end{aligned}
$$

because $p\left(t, \theta, \theta^{\prime}\right)$ and $P_{r}[\tau \leq t]=P_{x}[\sigma \leq t]$ are strictly positive by Lemma 3.2 .

Q.E.D.

LEMma 3.4. If $f \in C(\bar{B})$ and $\alpha$ is positive, the function $x \rightarrow$ $E_{x}\left[\int_{0}^{o} e^{-\alpha t} f\left(x_{t}\right) d t\right]$ belongs to $C(B)$. If $g \in C(\partial B)$ and $\alpha$ is positive, the function $x \rightarrow E_{x}\left[e^{-\alpha \sigma} g\left(x_{\sigma}\right)\right]$ also belongs to $C(B)$.

Proof. We first prove the continuity of $E_{x}\left[e^{-\alpha \sigma} g\left(x_{\sigma}\right)\right]$. Evidently, the family of finite linear combinations of spherical harmonics $\left\{S_{n}^{\ell}\right\}$ is dense in $C(\partial B)$. When $g \equiv 1$ and $x=(r, \theta)(0 \leq r<q)$, it is clear that 


$$
E_{x}\left[e^{-\alpha \sigma} g\left(x_{\sigma}\right)\right]=E_{r}\left[e^{-\alpha \tau}\right] .
$$

As is well known, the function $E_{r}\left[e^{-\alpha \tau}\right]$ of $r$ is continuous on [0, $]$.

When $g=S_{n}^{\ell}(n \geq 1)$, two cases must be distinguished. In case $x=$ $(0, \theta)$, it holds that

$$
E_{x}\left[e^{-\alpha \sigma} g\left(x_{\sigma}\right)\right]=E_{0}\left[e^{-\alpha \tau}\right] \int_{S^{d-1}} S_{n}^{\ell}(\theta) d \theta=0
$$

In case $x=(r, \theta)(0<r<q)$, we have

$$
\begin{aligned}
E_{x}\left[e^{-\alpha \sigma} g\left(x_{\sigma}\right)\right] & =E_{r} \times E_{\theta}\left[e^{-\alpha \tau} S_{n}^{\ell}\left(\theta\left(\ell_{\tau}\right)\right)\right] \\
& =E_{r}\left[e^{-\alpha \tau} \int_{S^{d-1}} p\left(\ell_{\tau}, \theta, \theta^{\prime}\right) S_{n}^{\ell}\left(\theta^{\prime}\right) d \theta^{\prime}\right] \\
& =E_{r}\left[e^{-\alpha \tau-r_{n} \ell_{\tau}}\right] S_{n}^{\ell}(\theta) .
\end{aligned}
$$

Now we consider the non-singular diffusion process $\tilde{R}=\left(\tilde{r}_{t}, \tilde{P}_{r}\right)$ on $(0, q]$ whose infinitesimal operator is $D_{m} D_{s}^{+}-\gamma_{n} r^{-2}$ with boundary conditions that $u(0)=0$ and $D_{s}^{-} u(q)=0$. Recall that the infinitesimal operator of the process $R$ is $D_{m} D_{s}^{+}$with boundary condition that $D_{s}^{-} u(q)=0$. We denote by $\tilde{\tau}$ the first hitting time to $q$ of $\tilde{R}$. We note that $\tilde{E}_{r}\left[e^{-\alpha \tau}\right]=$ $E_{r}\left[e^{-\alpha \tau-\gamma_{n} \ell_{\tau}}\right]$ [6]. Since the process $\tilde{R}$ is non-singular on $(0, q]$ and since $r=0$ is neither exit nor entrance, $E_{r}\left[e^{-\alpha \tau-r_{n} \ell_{\tau}}\right]$ is continuous on $(0, q]$ and converges to zero as $r$ decreases to zero [6]. Thus the second assertion has been proved. Let $C$ be all continuous functions on $[0, q]$ and $C_{0}$ be all continuous functions on $[0, q]$ vanishing in the neighbourhood of zero. We note that the family $\left\{\sum_{n=0}^{p} \sum_{\ell} e_{n}^{\ell}(r) S_{n}^{\ell}(\theta) \mid e_{0}^{0} \in C, e_{n}^{\ell} \in C_{0}, p\right.$ non-negative integer $\}$ is dense in $C(\bar{B})$ [12]. If $f \in C$, it holds that

$$
E_{x}\left[\int_{0}^{\sigma} e^{-\alpha t} f\left(x_{t}\right) d t\right]=E_{r}\left[\int_{0}^{\tau} e^{-\alpha t} f\left(r_{t}\right) d t\right]
$$

As is well known, the right side is continuous in $r$ on $[0, q]$. If $f=$ $h S_{n}^{\ell}\left(h \in C_{0}\right.$ and $\left.n \geq 1\right)$, two cases must be distinguished. In case $x=$ $(0, \theta)$, we can easily see that $E_{x}\left[\int_{0}^{o} e^{-\alpha t} f\left(x_{t}\right) d t\right]$ vanishes. In case $x=$ $(r, \theta)(0<r<q)$, it holds that

$$
\begin{aligned}
E_{x}\left[\int_{0}^{\sigma} e^{-\alpha t} f\left(x_{t}\right) d t\right] & =E_{r} \times E_{\theta}\left[\int_{0}^{\tau} e^{-\alpha t} h\left(r_{t}\right) S_{n}^{\ell}\left(\theta\left(\ell_{t}\right)\right) d t\right] \\
& =E_{r}\left[\int_{0}^{\tau} e^{-\alpha t-r_{n} \ell t} h\left(r_{t}\right) d t\right] S_{n}^{\ell}(\theta),
\end{aligned}
$$




$$
\begin{aligned}
E_{r}\left[\int_{0}^{\tau} e^{-\alpha t-\gamma_{n} \ell_{t}} h\left(r_{t}\right) d t\right] & =E_{r}\left[\int_{0}^{\infty} e^{-\alpha t-\gamma_{n} \ell_{t}} h\left(r_{t}\right) d t\right] \\
& -E_{r}\left[e^{-\alpha \tau-\gamma_{n} \ell_{\tau}}\right] E_{q}\left[\int_{0}^{\infty} e^{-\alpha t-\gamma_{n} \ell_{t}} h\left(r_{t}\right) d t\right] .
\end{aligned}
$$

But $E_{r}\left[\int_{0}^{\infty} e^{-\alpha t-\gamma_{n} \ell_{t}} h\left(r_{t}\right) d t\right]$ is continuous on $(0, q]$ and converges to zero as $r$ decreases to zero because it is an element in the range of the resolvent operator of the process $\tilde{R}$ which induces a continuous semi-group on $\hat{C}((0, q])$. We have already shown that $E_{r}\left[e^{-\alpha \tau-\gamma_{n} \ell_{t}}\right]$ also has the same property. Now the first assertion of the lemma follows.

Q.E.D.

LEMMA 3.5. Both transition probabilities of the process $X$ and of the part process $X_{B}$ are equivalent to the Lebesgue measure.

Proof. Both transition probabilities of the process $R$ and of the part process $R_{[0, q)}$ have positive densities with respect to the speed measure $d m(r)$ [6]. The transition density $p\left(t, \theta, \theta^{\prime}\right)$ of the spherical Brownian motion is also positive. The lemma follows from these facts.

Q.E.D.

LEMMA 3.6. Set $\varphi(x)=a l_{A \cap B}(x)+c l_{C}(x)$. Then $\varphi(x) d x$ is an invariant measure of the process $X$ on $\bar{B}$.

Proof. As is known, $d m(r)$ is an invariant measure of the process $R$ ([6] or [7]). Recall that $R$ is the radial part of $X$. In addition the transition density of the spherical Brown motion is symmetric. Thus if $F \in \mathscr{B}((0, q])$ and $G \in \mathscr{B}\left(S^{d-1}\right)$, it follows that

$$
\begin{aligned}
\int_{B} E_{x}\left[\left(l_{F} \cdot l_{G}\left(x_{t}\right)\right] \varphi(x) d x\right. & =\int_{(0, q] \times S^{d-1}} E_{r} \times E_{\theta}\left[l_{F}\left(r_{t}\right) l_{G}\left(\theta\left(\ell_{t}\right)\right)\right] d m(r) \times d \theta \\
& =\int_{(0, q]} l_{F} d m \int_{S^{d-1}} l_{G} d \theta .
\end{aligned}
$$

This proves the lemma.

Q.E.D.

\section{Proof fo Theorem 1}

Notations are the same as in Section 1. Let $\psi$ be a parallel translation in the $d$-dimensional Eucleadian space $E^{d}$ such that $\psi(\bar{D}) \cap \bar{D}$ is empty. $S$ denotes $B_{0} \cup_{i=1}^{n} \psi\left(B_{i}\right)$ and $\psi\left(X_{B_{i}}^{i}\right)(i=1, \cdots, n)$ the $\psi$ image of the part process $X_{B_{i}}^{i}$. Consider a following motion of a particle on $S$. The particle situated, for instance on $B_{0}$ when $t$ is zero, moves 
according to the law $X_{B_{0}}^{0}$ until it hits $\partial C$. When it hits $x \in \partial C_{i}$, it jumps up instantaneously to $\psi(x) \in \psi\left(B_{i}\right)$ and moves according to the law $\psi\left(X_{B_{i}}^{i}\right)$ until it hits $\psi\left(\partial B_{i}\right)$. When it hits $x \in \psi\left(\partial B_{i}\right)$, it jumps down instantaneously to $\psi^{-1}(x) \in B_{0}$ and moves according to the law $X_{B_{0}}^{0}$ and so on. We can describe this motion as a right-continuous strong Markov process on $S$. For the details we refer to [5] or [10]. This process turns out to be conservative on account of (1) in Lemmas 2.2 and 3.2. Now let $\gamma$ be the mapping from $S$ onto $\bar{D}$;

$$
\begin{array}{ll}
\gamma(x)=x & \text { if } x \in B_{0} \\
\gamma(x)=y & \text { if } x \in \psi(y) .
\end{array}
$$

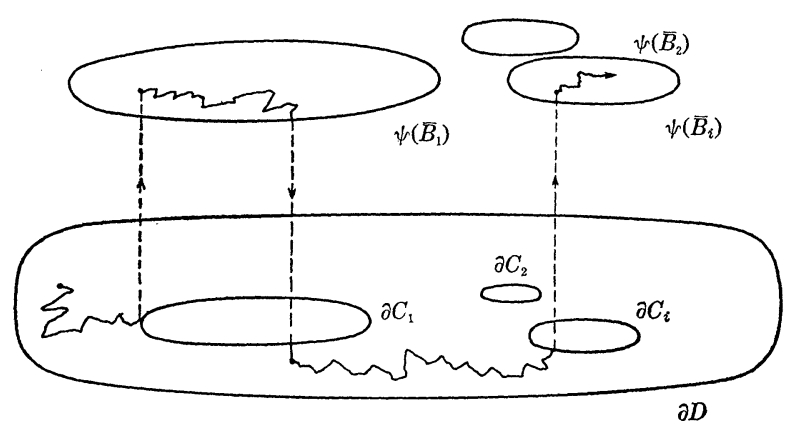

Figure 3

We project to $\bar{D}$ the process on $S$ by the mapping $\gamma$ to obtain a diffusion process on $\bar{D}$ (Theorem 10.13 in [2]). In this step it is essential that every part process $X_{B_{0} \cap B_{i}}^{0}$ is equivalent to $X_{B_{0} \cap B_{i}}^{i}(i=1, \cdots, n)$. The fact follows immediately from Lemmas 2.1 and 3.1. We can easily check that the process on $\bar{D}$ thus obtained satisfies the condition in Theorem 1 . In this way we can show the existence of a process in Theorem 1. Above we only outlined the procedure of the proof of the existence, because the rigorous description requires much space. We will establish the following two lemmas to complete the proof of Theorem 1.

LEMMA 4.1. Let $X=\left(X_{t}, P_{x}\right)$ be a right continuous strong Markov process on $\bar{D}$ satisfying the condition in Theorem 1 . If $f \in C(\bar{D})$ and $\alpha$ is positive, the function $x \rightarrow E_{x}\left[\int_{0}^{\infty} e^{-\alpha t} f\left(X_{t}\right) d t\right]$ belongs to $C(\bar{D})$.

Proof. Let $\tau$ be the first exit time from $B_{0}$ and assume that $x \in B_{0}$. It holds that 


$$
\begin{aligned}
E_{x}\left[\int_{0}^{\infty} e^{-\alpha t} f\left(X_{t}\right) d t\right] & =E_{x}\left[\int_{0}^{\tau} e^{-\alpha t} f\left(X_{t}\right) d t\right]+E_{x}\left[e^{-\alpha \tau} E_{x_{\tau}}\left[\int_{0}^{\infty} e^{-\alpha t} f\left(X_{t}\right) d t\right]\right] \\
& =E_{x}^{0}\left[\int_{0}^{\tau} e^{-\alpha t} f\left(X_{t}\right) d t\right]+E_{x}^{0}\left[e^{-\alpha \tau} E_{x_{\tau}}\left[\int_{0}^{\infty} e^{-\alpha t} f\left(X_{t}\right) d t\right]\right]
\end{aligned}
$$

The last two terms are continuous on $B_{0}$ due to Lemma 2.4. In particular, $E_{x}\left[\int_{0}^{\infty} e^{-\alpha t} f\left(X_{t}\right) d t\right]$ is continuous on $\partial B$. Using the first exit time from $B_{i}(i=1, \cdots, n)$, we can prove that $E_{x}\left[\int_{0}^{\infty} e^{-\alpha t} f\left(X_{t}\right) d t\right]$ is continuous on $B_{i}$ in the same way applying Lemma 3.4 .

As every process $X^{i}(i=0,1, \cdots, n)$ induces a continuous Feller semi-group, we denote by $\mathscr{A}_{i}$ and $D\left(\mathscr{A}_{i}\right)$ its infinitesimal operator and its domain. We need some other notations:

$$
\begin{aligned}
D_{i}^{K}= & \left\{f \in C(\bar{D}) \mid f \text { is an extension of an element in } D\left(\mathscr{A}_{i}\right)\right. \text { such that } \\
& \left.\quad \text { supp } f \subset B_{i}\right\}(i=0,1, \cdots, n), \\
\tilde{D}= & \left\{f=\sum_{i=0}^{n} f_{i} \mid f_{i} \in D_{i}^{K}(i=0,1, \cdots, n)\right\},
\end{aligned}
$$

$T_{t}$ is the semi-group on $B(\bar{D})$ induced by the process $X$ in Lemma 4.1, $L_{0}=\left\{f \in B(\bar{D}) \mid \lim _{t \rightarrow 0}\left\|T_{t} f-f\right\|=0\right\}$,

$\hat{\mathscr{A}}$ and $D(\hat{\mathscr{A}})$ are the infinitesimal operator of the semi-group $T_{t}$ on $L_{0}$ and its domain respectively.

LEMMA 4.2. Assume that the process $X=\left(X_{t}, P_{x}\right)$ is the same as in Lemma 4.1. The process $X$ induces a continuous Feller semi-group on $C(\bar{D})$. We denote by $\mathscr{A}$ and $D(\mathscr{A})$ its infinitesimal operator and its domain respectively. It holds that $l \in \tilde{D} \subset D(\mathscr{A})$ and that $\mathscr{A} l=0$. Therefore the process $X$ is necessarily conservative.

Proof. We first prove that $D_{0}^{K} \subset \hat{D}$. Assume that $f \in D_{0}^{K}$ and let $K_{i 0}$ and $K_{0 i}$ be open balls such that $C_{i} \subset K_{i 0} \subset K_{0 i} \subset B_{i}$ with the common center $\varepsilon_{i}(i=1, \cdots, n)$ and that supp $f \subset \bigcap_{i=1}^{n} K_{0 i}^{c}$. We denote by $\tau$ and $\sigma_{i}^{\prime}(i=1, \cdots, n)$ the first exit times from $B_{0}$ and from $K_{0 i}$ respectively. If $x \in K_{00} \equiv \bigcap_{i=1}^{n} K_{i 0}^{c}$, we have

$$
\begin{aligned}
E_{x}\left[f\left(X_{t}\right)\right] & =E_{x}\left[f\left(X_{t}\right): t<\tau\right]+E_{x}\left[f\left(X_{t}\right): \tau \leq t\right] \\
& =E_{x}^{0}\left[f\left(x_{t}\right)\right]-E_{x}^{0}\left[f\left(x_{t}\right): \tau \leq t\right]+E_{x}\left[f\left(X_{t}\right): \tau \leq t\right] .
\end{aligned}
$$

Hence it follows that 


$$
\begin{aligned}
& \left\|\frac{E_{x}\left[f\left(X_{t}\right)\right]-f(x)}{t}-\mathscr{A}_{0} f\right\|_{K_{00}} \leq\left\|\frac{E_{x}^{0}\left[f\left(x_{t}\right)\right]-f(x)}{t}-\mathscr{A}_{0} f\right\|_{K_{00}} \\
& \quad+2\|f\| \sup _{x \in K_{00}} \frac{P_{x}^{0}[\tau \leq t]}{t} .
\end{aligned}
$$

If $x \in K_{i 0}$, it holds that $f(x)=\mathscr{A}_{0} f(x)=0$ and that $E_{x}\left[f\left(X_{t}\right)\right]=$ $E_{x}\left[f\left(X_{t}\right): \sigma_{i}^{\prime} \leq t\right]$. Thus it follows that

$$
\left\|\frac{E_{x}\left[f\left(X_{t}\right)\right]-f(x)}{t}-\mathscr{A}_{0} f\right\|_{K i_{0}} \leq\|f\| \sup _{x \in K_{i 0}} \frac{P_{x}^{i}\left[\sigma_{i}^{\prime} \leq t\right]}{t} .
$$

Applying Lemmas 2.2 and 3.2 to (4.1) and (4.2) we conclude that $D_{0}^{K} \subset$ $D(\hat{\mathscr{A}})$ and that $\hat{\mathscr{A}} f=\mathscr{A}_{0} f$ if $f \in D_{0}^{K}$. By the similar argument, we see that $\tilde{D} \subset D(\hat{\mathscr{A}})$. Since $\tilde{D}$ is dense in $C(\bar{D}), C(\bar{D})$ is contained in $L_{0}$ (as to the domain $D\left(\mathscr{A}_{0}\right)$ we refer to [11]). Lemma 4.1 tells us that the resolvent operators of the semi-group $T_{t}$ on $L_{0}$ map $C(\bar{D})$ into itself, while $\tilde{D}$ is dense in $C(\bar{D})$. By the general theory of the semi-group, we conclude that the restriction of the semi-group $T_{t}$ to $C(\bar{D})$ is a continuous Feller semi-group. Apparently it holds that $l \in \tilde{D} \subset D(\mathscr{A})$ and that $\mathscr{A}_{i} l(x)=0(i=0,1, \cdots, n)$. On account of the local property of the infinitesimal operator, it follows that $\mathscr{A} l(x) \equiv 0$.

Q.E.D.

We observe that all characteristic operators of right-continuous strong-Markov process satisfying the condition in Theorem 1 coincide. Since such processes induce continuous Feller semi-groups, their infinitesimal operators are certain restrictions of characteristic operators (Theorem 5.5 in [2]). Consequently all such infinitesimal operators coincide. Namely, they all induce a common Feller semi-group on $C(\bar{D})$. This completes the proof of Theorem 1 .

\section{Proof of Theorem 2}

Notations are the same as in Section 1 and $X=\left(X_{t}, P_{x}\right)$ denotes the diffusion process specified in Theorem 1.

LEMMA 5.1. The probability measure $\mu$ in (1.3) is an invariant measure of the process $X$.

Proof. We will show that

$$
\int_{D} E_{x}\left[f\left(X_{t}\right)\right] \mu(d x) \leq \int_{D} f d \mu, \quad \text { if } f \in B(\bar{D}) \text { and } f \geq 0
$$


from which the invariance of $\mu$ immediately follows because the process $X$ is conservative. Let $K_{i 0}, K_{0 i}, H_{i 0}$ and $H_{0 i}$ be open balls such that $C_{i}$ $\subset K_{i 0} \subset K_{0 i} \subset H_{i 0} \subset H_{0 i} \subset B_{i}$ with the common center $\varepsilon_{i}(i=1, \cdots, n)$. We note that the partition of unity enables us to represent the nonnegative bounded function $f$ as a sum of non-negative functions $f_{i}^{\prime} s$ such that $\operatorname{supp} f_{0} \subset \bigcap_{i=1}^{n} K_{0 i}^{c}$ and that $\operatorname{supp} f_{i} \subset \bar{H}_{i 0}(i=1, \cdots, n)$. Assume that supp $f \subset \cap_{i=1}^{n} K_{0 i}^{c}$ and let $\tau$ and $\sigma_{i}^{\prime}(i=1, \cdots, n)$ be the first exit times from $B_{0}$ and $K_{0 i}$ respectively. If $x \in K_{00} \equiv \bigcap_{i=1}^{n} K_{i 0}^{c}$, it holds that

$$
\begin{aligned}
E_{x}\left[f\left(X_{t}\right)\right] & =E_{x}\left[f\left(X_{t}\right): t<\tau\right]+E_{x}\left[f\left(X_{t}\right): \tau \leq t\right] \\
& \leq E_{x}\left[f\left(X_{t}\right): t<\tau\right]+\|f\| \sup _{x \in K_{00}} P_{x}[\tau \leq t] .
\end{aligned}
$$

If $x \in K_{i 0}$, it holds that

$$
\begin{aligned}
E_{x}\left[f\left(X_{t}\right)\right] & =E_{x}\left[f\left(X_{t}\right): t<\sigma_{i}^{\prime}\right]+E_{x}\left[f\left(X_{t}\right): \sigma_{i}^{\prime} \leq t\right] \\
& \leq\|f\| \sup _{x \in K_{i 0}} P_{x}\left[\sigma_{i}^{\prime} \leq t\right] .
\end{aligned}
$$

Since every part process $X_{B_{i}}$ is equivalent to $X_{B_{i}}^{i}(i=0,1, \cdots, n)$, we apply (2) Lemmas 2.2 and 3.2 and Lemma 2.6 to obtain

$$
\begin{aligned}
\int_{D} E_{x}\left[f\left(X_{t}\right)\right] \mu(d x) & =\sum_{i=0}^{n} \int_{K_{i 0}} E_{x}\left[f\left(X_{t}\right)\right] \mu(d x) \\
& \leq \int_{B_{0}} E_{x}\left[f\left(X_{t}\right): t<\tau\right]+\|f\| \sum_{i=0}^{n} \sup _{x \in K_{i 0}} P_{x}\left[\sigma_{i}^{\prime} \leq t\right] \\
& \leq \int_{B_{0}} f d \mu+\|f\| o(t)
\end{aligned}
$$

where $\sigma_{0}^{\prime}$ denotes $\tau$. Next assume that supp $f \subset \bar{H}_{i_{0}}$ ( $i$ to be fixed). Let $\tau^{\prime}$ and $\sigma_{i}(i=1, \cdots, n)$ be the first exit times from the interior of $\bigcap_{i=1}^{n} H_{i 0}^{c}$ and from $B_{i}$ respectively. In the same way as above we obtain

$$
\int_{D} E_{x}\left[f\left(X_{t}\right)\right] \mu(d x) \leq \int_{B_{i}} f d \mu+\|f\| o(t)
$$

In this case we must apply (1) in Lemmas 2.2 and 3.2 and Lemma 3.6. Thus for any non-negative function $f$ in $B(\bar{D})$, it holds that

$$
\int_{D} E_{x}\left[f\left(X_{t}\right)\right] \mu(d x) \leq \int_{D} f d \mu+\|f\| o(t)
$$

We observe that $o(t)$ does not depend on the function $f$. If $m$ is a large integer, it follows that 


$$
\begin{aligned}
\int_{D} E_{x}\left[f\left(X_{t}\right)\right] \mu(d x) & =\int_{D} E_{x}\left[E_{X_{t / m}}\left[f\left(X_{((m-1) / m) t}\right)\right]\right] \mu(d x) \\
& \leq \int_{D} E_{x}\left[f\left(X_{((m-1) / m) t}\right)\right] \mu(d x)+\|f\| o\left(\frac{t}{m}\right) \\
& \leq \int_{D} f d \mu+\|f\| o\left(\frac{t}{m}\right) m
\end{aligned}
$$

Since $m$ is arbitrary, we obtain (5.1).

Q.E.D.

LEMMA 5.2. The transition probability $\left\{P_{t}(x, \cdot)\right\}$ of the process $X$ is equivalent to the Lebesgue measure $\nu$, when $t$ is positive.

Proof. We may assume that the process $X$ is standard [2]. We define two sequences of Markov times $\left\{\sigma_{n}\right\}$ and $\left\{\tau_{n}\right\}$ by

$$
\begin{array}{ll}
\sigma_{0}=\text { the first hitting time to } \partial B, & \tau_{0}=\text { the first hitting time to } \partial C, \\
\sigma_{1}=\tau_{0}+\theta_{\tau_{0}} \sigma_{0}, & \tau_{1}=\sigma_{1}+\theta_{\sigma_{1}} \tau_{0}, \\
\sigma_{n+1}=\sigma_{n}+\theta_{\sigma_{n}} \sigma_{1}, & \tau_{n+1}=\tau_{n}+\theta_{\tau_{n}} \tau_{1} \quad(n \geq 2) .
\end{array}
$$

Using Lemmas 2.2 and 3.2 it is easy to see that these Markov times are finite and that increase to infinity as $n$ increases. First we will show that $\nu\left(F^{\prime}\right)=0$ implies $P_{t}(x, f)=0$. Whenever $t$ is positive it follows from Lemmas 2.5 and 3.5 that $E_{x}\left[l_{F}\left(X_{t}\right): t<\tau_{0}\right]$ vanishes on $B_{0}$ and that $E_{x}\left[l_{F}\left(X_{t}\right): t<\sigma_{0}\right]$ vanishes on $C$. We note that $P_{x}\left[\tau_{0}\right.$ or $\left.\sigma_{0}=t\right]$ vanishes if $t$ is positive. Indeed, if $x \in B_{0}$, since the transition probability of the process $X^{0}$ is equivalent to $\nu$, we have

$$
P_{x}\left[\tau_{0}=t\right]=P_{x}^{0}\left[\tau_{0}=t\right] \leq P_{x}^{0}\left[x_{t} \in \partial C\right]=0 .
$$

On the same reason, $P_{x}\left[\tau_{0}=t\right]$ vanishes on every $C_{i}(i=1, \cdots, n)$. Since the radial part of $X^{i}$ is non-singular, $P_{x}^{i}\left[\tau_{0}=0\right]\left(=P_{x}\left[\tau_{0}=0\right]\right)$ is equal to 1. Similary $P_{x}\left[\sigma_{0}=t\right]$ vanishes on $\bar{D}$. By the induction on $n$, we see that $P_{x}\left[\sigma_{n}\right.$ or $\left.\tau_{n}=t\right]$ also vanishes on $\bar{D}$. For instance, it holds that

$$
P_{x}\left[\sigma_{1}=t\right]=P_{x}\left[\tau_{0}+\theta_{\tau_{0}} \sigma_{0}=t\right]=E_{x}\left[P_{X \tau_{0}}\left[s+\sigma_{0}=t\right]_{s=\tau_{0}}\right] \leq P_{x}\left[\tau_{0}=t\right] .
$$

Hence it follows that

$$
\begin{aligned}
P_{t}(x, F)= & E_{x}\left[l_{F}\left(X_{t}\right): t<\tau_{0}\right]+\sum_{n=0}^{\infty} E_{x}\left[l_{F}\left(X_{t}\right): \tau_{n} \leq t<\sigma_{n+1}\right] \\
& +\sum_{n=1}^{\infty} E_{x}\left[l_{F}\left(X_{t}\right): \sigma_{n} \leq t<\tau_{n}\right]
\end{aligned}
$$




$$
\begin{aligned}
= & E_{x}\left[l_{F}\left(X_{t}\right): t<\tau_{0}\right]+\sum_{n=0}^{\infty} E_{x}\left[E_{X_{\tau_{n}}}\left[l_{F}\left(X_{t-s}\right): t-s<\sigma_{0}\right]_{s=\tau_{n}}: \tau_{n} \leq t\right] \\
& +\sum_{n=0}^{\infty} E_{x}\left[E_{X \sigma_{n}}\left[l_{F}\left(X_{t-s}\right): t-s<\tau_{0}\right]_{s=\sigma_{n}}: \sigma_{n} \leq t\right] \\
\leq & \sum_{n=0}^{\infty} P_{x}\left[\tau_{n}=t\right]+\sum_{n=1}^{\infty} P_{x}\left[\sigma_{n}=t\right]=0 .
\end{aligned}
$$

Now we will show that the positivity of $\nu(F)$ implies that of $P_{t}(x, F)$ if $t$ is positive. Clearly it holds that

$$
\begin{aligned}
E_{x}\left[l_{F}\left(X_{t}\right)\right] \geq & E_{x}\left[l_{F}\left(X_{t}\right): t<\tau_{0}\right]+E_{x}\left[l_{F}\left(X_{t}\right): \tau_{0} \leq t<\sigma_{1}\right] \\
& +E_{x}\left[l_{F}\left(X_{t}\right): \sigma_{1} \leq t<\tau_{1}\right] .
\end{aligned}
$$

Using the Markov property and Lemmas 2.3, 2.5, 3.3 and 3.5, we can show that the right side is positive if $t$ and $\nu(F)$ are positive. The proof of the lemma is complete.

Q.E.D.

Lemma 5.3. Let $Y=\left(Y_{t}, \infty, M_{t}, M, P_{x}\right)$ be a Markov process on a semi-compact state space $E$ with an invariant probability measure $\nu$. If probability measures $\left\{P_{t}(x, \cdot) \mid(t, x) \in(0, \infty) \times E\right\}$ are equivalent, for any probability measures $m_{1}$ and $m_{2}$ on $(E, \mathscr{B}(E))$, it holds that

$$
\lim _{t \rightarrow \infty}\left\|P_{t}\left(m_{1}, \cdot\right)-P_{t}\left(m_{2}, \cdot\right)\right\|=0,
$$

in particular

$$
\lim _{t \rightarrow \infty}\left\|P_{t}\left(m_{1}, \cdot\right)-\nu(\cdot)\right\|=0
$$

Furthermore, $\nu$ is the unique invariant measure of the process $Y$.

Proof. We note that the measure $\nu$ is equivalent to $\left\{P_{t}(x, \cdot)\right\}$. Indeed, $\nu$ is absolutely continuous with respect to $\left\{P_{t}(x, \cdot)\right\}$, while $\nu(F)$ $=0$ implies $P_{t}(x, F)=0$. We will show that the Markov chain $\left(Y_{n}, P_{x}\right)$ satisfies Harris' condition; there exists a $\sigma$-finite measure $\lambda$ on $(E, \mathscr{B}(E)$ ) such that $P_{x}\left[\sum_{n=1}^{\infty} l_{F}\left(Y_{n}\right)=\infty\right]$ is identically equal to 1 so far as $\lambda(F)$ is positive. Consider a sub $\sigma$-algebra $\mathscr{D}$ of $\mathscr{B}(E)$;

$\mathscr{D}=\left\{F \in \mathscr{B}(E) \mid\right.$ There exists a $\tilde{F} \in \mathscr{B}(E)$ such that $\left.E_{x}\left[l_{F}\left(Y_{1}\right)\right]=l_{\tilde{F}}(x)(a, e, \nu)\right\}$.

By the above note, $\mathscr{D}$ has to be a trivial algebra with respect to the measure $\nu$. Thus the Markov chain with the initial distribution $\nu$ is ergodic (cf. Proposition V-6-3 in [13]). We set $\Gamma=\left\{\omega \mid \sum_{n=1}^{\infty} l_{F}\left(Y_{n}\right)=\infty\right\}$, 
where $F$ is not a null set of $\nu$. We must show that $P_{x}[\Gamma]$ is identically equal to 1 on $E$. On account of the ergodic theorem, we have

$$
\lim _{n \rightarrow \infty} \frac{1}{n} \sum_{i=1}^{n} l_{F}\left(Y_{i}\right)=\nu(F) \quad\left(\text { a.e. } P_{\nu}\right) \text {. }
$$

Hence $P_{\nu}(\Gamma)$ is equal to 1 , namely $P_{x}[\Gamma]$ is identical equal to 1 with respect to $\nu$. Since the measure $\nu$ and $\left\{P_{t}(x, \cdot)\right\}$ are equivalent, $P_{x}[\Gamma]$ is identically equal to 1 , as is to be shown. Evidently the chain $\left(Y_{n}, P_{x}\right)$ is aperiodic. Thus (5.2) holds if both $m_{1}$ and $m_{2}$ are Dirac measures [8]. Now by the same reasoning as M. Duflo and D. Revuz we obtain (5.2) (II.3 in [1]). Because the chain satisfies Harris' condition, the uniqueness of the invariant measure also follows [4]. $\quad$ Q.E.D.

We will show (1.5) to complete the proof of Theorem 2. We set $H_{t}=\mathscr{B}\left[X_{u}: u \geq t\right]$ i.e. the $\sigma$-algebra generated by all sets $\left\{\omega \mid X_{u} \in F\right\}$ $(F \in \mathscr{B}(E)$ and $u \geq t)$. We denote the $\sigma$-algebra $\bigcap_{t>0} H_{t}$ by $H$. Since the process $\hat{X}=\left(X_{t}, P_{x}\right)$ satisfies (1.4), not only the $\sigma$-algebra $H$ is trivial with respect to $P_{\nu}$ whatever the initial distribution $\nu$ may be, but also $P_{x}[\Gamma]$ is a constant if $\Gamma \in H$. Therefore to prove (1.5) it suffices to show that $P_{\mu}[\Gamma]$ is equal to 1 , where

$$
\Gamma=\left\{\omega \mid \lim _{t \rightarrow \infty} \frac{1}{t} \int_{0}^{t} f\left(X_{s}\right) d s=\int_{D} f d \mu\right\}
$$

Consider a sequence of random variables $\left\{F_{n}\right\} ; F_{n}(\omega)=\int_{n-1}^{n} f\left(X_{t}\right) d t$. On account of the ergodic theorem there exists a $H$-measurable function $F^{*}(\omega)$ such that

$$
\lim _{n \rightarrow \infty} \frac{1}{n} \sum_{i=1}^{n} F_{i}(\omega)=F^{*}(\omega) \quad\left(\text { a.e.P } P_{\mu}\right)
$$

Since the $\sigma$-algebra $H$ is trivial, $F^{*}(\omega)$ is equal to $\int_{D} f d \mu$ with respect to $P_{\mu}$. After the standard argument, we know that $P_{\mu}[\Gamma]$ is equal to 1 . Now Theorem 2 is established.

\section{REFERENCES}

[1] M. Duflo and D. Revuz: Propriétés asymptotiques des probabilités de transition des processus de Markov récurrents. Ann. Inst. Henri Poincaré, vol. 5, nº3 (1969), 233-244.

[ 2 ] E. B. Dynkin: Markov process, Springer-Verlag, Berlin, 1965. 
[ 3 ] I. V. Girsanov: Strongly-Feller processes. Theory of Prob. and its Appl., vol. 5 (1960), 5-24.

[4] T. E. Harris: The existence of stationary measures for certain Markov processes, Third Berkley Sympo., vol. 1 (1956), 113-125.

[ 5 ] N. Ikeda, T. Nagasawa and S. Watanabe: Branching Markov processes II. J. Math. Kyoto Univ. 8-3 (1968), 365-410.

[6] K. Ito and H. P. Mckean, Jr.: Diffusion processes and their sample paths, Springer-Verlag, Berlin, 1965.

[ 7 ] G. Maruyama and H. Tanaka: Some properties of one-dimensional processes. Mem. Fac. Sci. Kyushu Univ. A-11, 2 (1957), 117-141.

[ 8 ] S. Orey: Recurrent Markov chains, Pacific J. of Math. 9 (1959), 805-827.

[ 9 ] Seminar on Prob. vol. 5 (1960) (Japanese).

[10] Seminar on Prob. vol. 23-I (1966) (Japanese).

[11] K. Sato and T. Ueno: Multidimensional diffusion process and the Markov process on the boundary. J. Math. Kyoto Univ. 4 (1965), 526-606.

[12] A. D. Wentzell: On boundary conditions for multidimensional diffusion processes. Theory of Prob. and its Appl. vol. 4 (1959), 164-177.

[13] J. Neveu: Bases mathématiques du calcul des probabilités. Paris, Masson et Cie, 1964.

Nagoya University 